\title{
On Boundedness of Symmetrical Motions in the Three-Body Problem
}

\section{SP Sosnitskii}

Institute of Mathematics, Ukrainian National Academy of Sciences, Kyiv, Ukraine

\begin{abstract}
In the present paper, we study the three-body problem in the case where two bodies have equal masses, which imply the existence of a manifold of symmetric motions. We find conditions of existence of bounded symmetric motions. These conditions can be useful for elucidating those key circumstances that cause the existence of oscillating final evolutions. For the analysis of boundedness of motions, both the structure of the manifold of symmetrical motions and the integrals of energy and angular momentum are essential.
\end{abstract}

\section{Keywords}

Symmetrical motion, Hill stable pair, Distal motion, Elliptic keplerian motion

\section{Introduction}

Symmetric motions in the three-body problem are usually associated with the problem of existence of oscillating eventual evolutions considered in the well-known work by Sitnikov [1]. Although that work is mainly devoted to the restricted elliptic three-body problem, it gave impetus to further research of the general three-body problem [2-4]. All the more, the possibility of existence of oscillating motions within the framework of the general three-body problem was admitted by Chazy [5].

In this paper, continuing author's research [6], we expand their spectrum somewhat.

Turning to the consideration of symmetric motions, we will not immediately write down the equations of motion that correspond to the manifold of symmetric motions, but we will start from the general equations of motion of the three-body problem, giving them the desired shape already in the process of research.

So, in the study of symmetric motions, we will first of all rely on the basic equations of the threebody problem representing them in the form [7]:

$$
\begin{aligned}
& \rho_{1}^{\prime \prime}=\mu_{2} \frac{\rho_{2}-\rho_{1}}{\left|\rho_{12}\right|^{3}}+\mu_{3} \frac{\rho_{3}-\rho_{1}}{\left|\rho_{13}\right|^{3}}, \\
& \rho_{2}^{\prime \prime}=-\mu_{1} \frac{\rho_{2}-\rho_{1}}{\left|\rho_{12}\right|^{3}}+\mu_{3} \frac{\rho_{3}-\rho_{2}}{\left|\rho_{23}\right|^{3}}, \\
& \rho_{3}^{\prime \prime}=-\mu_{1} \frac{\rho_{3}-\rho_{1}}{\left|\rho_{13}\right|^{3}}-\mu_{2} \frac{\rho_{3}-\rho_{2}}{\left|\rho_{23}\right|^{3}}
\end{aligned}
$$

Where the prime symbol means differentiation with respect to

*Corresponding author: SP Sosnitskii, Institute of Mathematics, Ukrainian National Academy of Sciences, Ukraine

Accepted: December 04, 2020; Published: December 06, 2020

Copyright: (C) 2021 Sosnitskii SP. This is an open-access article distributed under the terms of the Creative Commons Attribution License, which permits unrestricted use, distribution, and reproduction in any medium, provided the original author and source are credited.

Sosnitskii. Int J Astronaut Aeronautical Eng 2020, 5:043

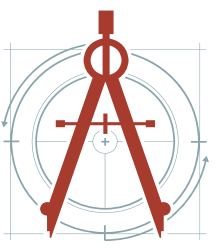




$$
\tau\left(\tau=t \sqrt{G M} / r_{0}^{\frac{3}{2}}\right) ; \mu_{i}=\mathrm{m}_{i} / M, M=m_{1}+m_{2}+m_{3} ; r_{0} \text { is a parameter that has the dimension of the }
$$

unit of length. In equations (1.1), we have $\boldsymbol{\rho}_{i}=\mathbf{r}_{i} / r_{0}, i=1,2,3$, where $r_{i}$ are the radius vectors of points in the inertial reference frame with the origin at the center of mass of $m_{i}$. The parameter $r_{o}$ having the dimension of the unit of length is included into the expression of $\tau$ in order to deal with dimensionless quantities since it is very convenient from the viewpoint of our subsequent study.

In what follows, we essentially use the conservative property of system (1.1), i.e. the existence of the energy integral

$$
\frac{1}{2} \sum_{i}^{3} \mu_{i} \boldsymbol{\rho}_{i}^{\prime 2}-\sum_{i<j} \frac{\mu_{i} \mu_{j}}{\left|\boldsymbol{\rho}_{i j}\right|}=h=\mathrm{const}
$$

and the vector integral of angular momentum

$$
\sum_{i}^{3} \mu_{1}\left(\rho_{i} \times \rho_{i}^{\prime}\right)=\mathrm{C} \text {. }
$$

We assume that $C \neq 0$.

Further, without loss of generality, we also assume that the equality

$$
\sum_{i}^{3} \mu_{i} \rho_{i}=0
$$

is satisfied; This means that the origin of the reference system is located at the center of mass of the material points (bodies).

\section{On the Manifold of Symmetric Motions of the Three-Body Problem}

As is shown in [6], if two masses are equal, then we arrive at the manifold of symmetric motions

$$
\begin{aligned}
& \rho_{12}^{\prime \prime}=-2 \mu \frac{\rho_{12}}{\left|\rho_{12}\right|^{3}}-\mu_{3} \frac{\rho_{12}}{\left.|| \rho_{13}\right|^{3} \mid} \\
& \rho_{3}^{\prime \prime}=-\frac{\rho_{3}}{\left|\rho_{13}\right|^{3}}
\end{aligned}
$$

Where $\left|\boldsymbol{\rho}_{13}\right|=\left|\boldsymbol{\rho}_{23}\right|, \mu_{1}=\mu_{2}=\mu, 2 \mu+\mu_{3}=1$, and the following equality is satisfied:

$$
\rho_{3}^{2}=\mu^{2}\left(-\rho_{12}^{2}+4 \rho_{13}^{2}\right)
$$

A distinctive feature of the manifold of symmetric motions of system (2.1) is the validity of equalities

$$
\rho_{12} \times \rho_{12}^{\prime}=C_{1}, \rho_{3} \times \rho_{3}^{\prime}=C_{2} \text {, }
$$

Where $C_{1}, C_{2}$ are constant vectors. This enables us to perform a qualitative study of system (2.1) given that

$$
v_{12}^{2}=\rho_{12}^{\prime 2}=\rho_{12}^{\prime 2}+\frac{\left|\boldsymbol{\rho}_{12} \times \rho_{12}^{\prime}\right|^{2}}{\rho_{12}^{2}},
$$




$$
v_{3}^{2}=\rho_{3}^{\prime 2}=\rho_{3}^{\prime 2}+\frac{\left|\rho_{3} \times \rho^{\prime}\right|^{2}}{\rho_{3}^{2}}
$$

and reduce it to the following system with two degrees of freedom:

$$
\begin{aligned}
& \rho_{12}^{2 \prime \prime}=2 v_{12}^{2}-\frac{4 \mu}{\rho_{12}}-2 \mu_{3} \frac{\rho_{12}^{2}}{\rho_{13}^{3}}, \\
& \rho_{3}^{2 \prime \prime}=2 v_{3}^{2}-2 \frac{\rho_{3}^{2}}{\rho_{13}^{3}}
\end{aligned}
$$

If $C_{2}=\mathbf{0}$, then system (2.6) admits a motion, for which the body having the mass $\mu_{3}$ oscillates along an axis passing through the center of mass of the system and perpendicular to the plane of movement of the other two bodies with equal masses. It is the case that was considered by Sitnikov [1].

We arrived at system (2.6) based on equations (1.1). We now use the distance equations [8], taking into account the relations obtained in this article:

$$
\rho_{12}^{\prime} \rho_{13}-\rho_{12} \rho_{13}^{\prime}=\rho_{12}^{\prime} \rho_{23}-\rho_{12} \rho_{23}^{\prime}=\rho_{13}^{\prime} \rho_{23}-\rho_{13} \rho_{23}^{\prime}
$$

If we notice that in the case under consideration

$$
\rho_{12}^{\prime} \rho_{13}-\rho_{12} \rho_{13}^{\prime}=\rho_{12}^{\prime} \rho_{23}-\rho_{12} \rho_{23}^{\prime}=\rho_{13}^{\prime} \rho_{23}-\rho_{13} \rho_{23}^{\prime}=0
$$

Which follows from equations (1.1) in the form

$$
\begin{aligned}
& \rho_{12}^{\prime \prime}=-\left(1-\mu_{3}\right) \frac{\rho_{12}}{\left|\rho_{12}\right|^{3}}+\mu_{3}\left(-\frac{\rho_{13}}{\left|\rho_{13}\right|^{3}}+\frac{\rho_{23}}{\left|\rho_{22}\right|^{3}}\right), \\
& \rho_{13}^{\prime \prime}=-\left(1-\mu_{2}\right) \frac{\rho_{13}}{\left|\rho_{13}\right|^{3}}-\mu_{2}\left(-\frac{\rho_{12}}{\left|\rho_{12}\right|^{3}}+\frac{\rho_{23}}{\left|\rho_{23}\right|^{3}}\right), \\
& \rho_{23}^{\prime \prime}=-\left(1-\mu_{1}\right) \frac{\rho_{23}}{\left|\rho_{23}\right|^{3}}+\mu_{1}\left(\frac{\rho_{12}}{\left|\rho_{12}\right|^{3}}-\frac{\rho_{13}}{\left|\rho_{23}\right|^{3}}\right),
\end{aligned}
$$

and, in turn, the equalities

$$
\begin{aligned}
& \left(\boldsymbol{\rho}_{12} \boldsymbol{\rho}_{13}\right)^{\prime}=\boldsymbol{\rho}_{12}^{\prime} \boldsymbol{\rho}_{13}+\boldsymbol{\rho}_{12} \boldsymbol{\rho}_{13}^{\prime}=\frac{1}{2}\left(\rho_{12}^{2}+\rho_{13}^{2}-\rho_{23}^{2}\right)^{\prime}=\frac{1}{2}\left(\rho_{12}^{2}\right)^{\prime}, \\
& \left(\boldsymbol{\rho}_{12} \boldsymbol{\rho}_{23}\right)^{\prime}=\boldsymbol{\rho}_{12}^{\prime} \boldsymbol{\rho}_{23}+\boldsymbol{\rho}_{12} \boldsymbol{\rho}_{23}^{\prime}=\frac{1}{2}\left(-\rho_{12}^{2}+\rho_{13}^{2}-\rho_{23}^{2}\right)^{\prime}=\frac{1}{2}\left(-\rho_{12}^{2}\right)^{\prime}, \\
& \left(\boldsymbol{\rho}_{13} \boldsymbol{\rho}_{23}\right)^{\prime}=\boldsymbol{\rho}_{13}^{\prime} \boldsymbol{\rho}_{23}+\boldsymbol{\rho}_{13} \boldsymbol{\rho}_{23}^{\prime}=\frac{1}{2}\left(-\rho_{12}^{2}+\rho_{13}^{2}-\rho_{23}^{2}\right)^{\prime}=\frac{1}{2}\left(-\rho_{12}^{2}+2 \rho_{13}^{2}\right)^{\prime}
\end{aligned}
$$

are valid, then we obtain the manifold of symmetric motions in the form

$$
\rho_{12}^{2 \prime \prime}=2 v_{12}^{2}-\frac{4 \mu}{\rho_{12}}-2 \mu_{3} \frac{\rho_{12}^{2}}{\rho_{13}^{3}},
$$




$$
\begin{aligned}
& \rho_{13}^{2 \prime \prime}=2 v_{13}^{2}-\frac{2}{\rho_{13}}+\mu\left(-\frac{1}{\rho_{12}}+\frac{\rho_{12}^{2}}{\rho_{13}^{3}}\right), \\
& E_{12}^{\prime}=\mu_{3} \rho_{12}^{2 \prime}\left(\frac{1}{\rho_{12}^{3}}-\frac{1}{\rho_{13}^{3}}\right), \\
& E_{13}^{\prime}=-\frac{\mu}{2} \rho_{12}^{2 \prime}\left(\frac{1}{\rho_{12}^{3}}-\frac{1}{\rho_{13}^{3}}\right),
\end{aligned}
$$

Where,

$$
E_{12}=v_{12}^{2}-\frac{2}{\rho_{12}}, E_{13}=v_{13}^{2}-\frac{2}{\rho_{13}} \text {. }
$$

Taking into account equalities (2.2) - (2.5), as well as the equality

$$
v_{3}^{2}=-\mu^{2} v_{12}^{2}+4 \mu^{2} v_{13}^{2} \text {, }
$$

We see that this is also a system with two degrees of freedom. In particular, its first two equations form a closed system. The remaining two equations are its consequence.

The energy integral for system (2.11) has the form

$$
\mu^{2} E_{12}+2 \mu \mu_{3} E_{13}=2 h
$$

Further, we restrict ourselves to such symmetric motions of system (2.11) (or (2.6)) that belong to the set

$$
\Omega=\left\{\left(\rho, \rho^{\prime}\right): T-U=h<0\right\}
$$

\section{On Stationary Symmetric Motions}

Based on the structure of the manifold of symmetric motions both in the form (2.6) and in the form (2.11), we see that the stationary symmetric motions, i.e. movements for which the distances $\rho_{12}, \rho_{13}, \rho_{3}$ are constant, correspond to the equilibrium positions of the systems of equations (2.6) or (2.11) respectively. Next, we dwell on the system (2.11). Taking into account the energy integral (2.14), we rewrite it in the form

$$
\begin{aligned}
& \rho_{12}^{2^{\prime \prime}}=2\left(\frac{\left(\rho_{12}^{2 \prime}\right)^{2}}{4 \rho_{12}^{2}}+\frac{\left|\mathrm{C}_{1}\right|^{2}}{\rho_{12}^{2}}\right)-\frac{4 \mu}{\rho_{12}}-2 \mu_{3} \frac{\rho_{12}^{2}}{\rho_{13}^{3}}, \\
& \rho_{13}^{2^{\prime \prime}}=\frac{2 h}{\mu \mu_{3}}-\frac{\mu}{\mu_{3}}\left(\frac{\left(\rho_{12}^{2 \prime}\right)^{2}}{4 \rho_{12}^{2}}+\frac{\left|\mathrm{C}_{1}\right|^{2}}{\rho_{12}^{2}}\right)+\frac{\mu\left(2-\mu_{3}\right)}{\mu_{3} \rho_{12}}+\frac{2}{\rho_{13}}+\mu \frac{\rho_{12}^{2}}{\rho_{13}^{3}} .
\end{aligned}
$$

To determine the equilibrium positions, we arrive at the equations

$$
2\left(\frac{\left(\rho_{12}^{2 \prime}\right)^{2}}{4 \rho_{12}^{2}}+\frac{\left|\mathrm{C}_{1}\right|^{2}}{\rho_{12}^{2}}\right)-\frac{4 \mu}{\rho_{12}}-2 \mu_{3} \frac{\rho_{12}^{2}}{\rho_{13}^{3}}=0,
$$


$\frac{2 h}{\mu \mu_{3}}-\frac{\mu}{\mu_{3}}\left(\frac{\left(\rho_{12}^{2 \prime}\right)^{2}}{4 \rho_{12}^{2}}+\frac{\left|\mathrm{C}_{1}\right|^{2}}{\rho_{12}^{2}}\right)+\frac{\mu\left(2-\mu_{3}\right)}{\mu_{3} \rho_{12}}+\frac{2}{\rho_{13}}+\mu \frac{\rho_{12}^{2}}{\rho_{13}^{3}}=0$.

Given the fact that in the equilibrium position $\rho_{12}^{2 \prime}=0$, we rewrite Eqs. (3.2) in the form

$$
\begin{aligned}
& 2\left(\frac{c^{2}}{\rho_{12}^{4}}\right)-\frac{4 \mu}{\rho_{12}^{3}}-2 \mu_{3} \frac{1}{\rho_{13}^{3}}=0, \\
& \frac{2 h}{\mu \mu_{3}} \frac{1}{\rho_{12}^{2}}-\frac{\mu}{\mu_{3}}\left(\frac{c^{2}}{\rho_{12}^{4}}\right)+\frac{\mu\left(2-\mu_{3}\right)}{\mu_{3}} \frac{1}{\rho_{12}^{3}}+\frac{1}{\rho_{12}^{2}} \frac{2}{\rho_{13}}+\mu \frac{1}{\rho_{13}^{3}}=0,
\end{aligned}
$$

Where, $c^{2}=\left|\mathrm{C}_{1}\right|^{2}$. Thus, we have a system of two nonlinear algebraic equations for the variables $1 / \rho_{12}$ and $1 / \rho_{13}$. It is required to prove that this system has at least one positive solution, given that $1 / \rho_{12}$ and $1 / \rho_{13}$ are positive values in their meaning.

Solving the first equation of system (3.3) with respect to $1 / \rho_{13}^{3}$ and substituting the resulting expression in the second equation of this system, we obtain

$$
\frac{1}{\rho_{12}^{2}}\left[2 \frac{h}{\mu \mu_{3}}+\frac{\mu}{\mu_{3}} \frac{1}{\rho_{12}}+\frac{2}{\rho_{13}}\right]=0
$$

Since in the case under consideration, $1 / \rho_{12}$ is positive, then on the basis of (3.4) we have

$$
\frac{1}{\rho_{13}}=-\frac{1}{\mu_{3}}\left[\frac{h}{\mu}+\frac{\mu}{2} \frac{1}{\rho_{12}}\right] \text {, }
$$

hence

$$
\frac{1}{\rho_{12}}<-\frac{2 h}{\mu^{2}}
$$

At the same time, on the basis of the first equation of system (3.3), we have

$$
\frac{1}{\rho_{12}}>\frac{2 \mu}{c^{2}}
$$

From the compatibility condition for inequalities (3.6) and (3.7) we obtain

$$
h c^{2}+\mu^{3}<0
$$

Now we substitute the value $1 / \rho_{13}$, which is expressed by the right-hand side of equality (3.5), into the first equation of system (3.3). As a result, we arrive at the equation

$$
8 c^{2} \mu^{3} \mu_{3}^{2} x^{4}+\mu^{4}\left(\mu^{2}-16 \mu_{3}^{2}\right) x^{3}+6 \mu^{4} h x^{2}+12 \mu^{2} h^{2} x+8 h^{3}=0,
$$

Where, $x=1 / \rho_{12}$.

Consider the left side of equation (3.9), which we denote by $P_{4}(x)$, for $x=2 \mu / c^{2}$ and $x=-2 h / \mu^{2}$, respectively. As a result, we get 


$$
\begin{aligned}
& P_{4}\left(\frac{2 \mu}{c^{2}}\right)=\frac{8}{c^{6}}\left(h c^{2}+\mu^{3}\right)^{3}, \\
& P_{4}\left(-\frac{2 h}{\mu^{2}}\right)=128 \frac{\mu_{3}^{2} h^{3}}{\mu^{5}}\left(h c^{2}+\mu^{3}\right),
\end{aligned}
$$

As can be seen from equalities (3.10) and (3.11), when passing from the value $x=2 \mu / c^{2}$ to the value $x=-2 h / \mu^{2}$, the polynomial $P_{4}(x)$, changes sign. Therefore, equation (3.9) has a positive root, which, given (3.5), indicates the presence of a positive solution to system (3.3).

Thus, equalities (3.6), (3.7), (3.10) and (3.11) allow us to obtain the following

Assertion 1. The manifold of symmetric motions (2.11) (or (2.6)) admits stationary motions (for $h<0$ and $C_{2} \neq 0$ ), if and only if the condition is satisfied:

$$
h c^{2}+\mu^{3}<0 \text {. }
$$

Necessity, as we could see above, follows from inequalities (3.6) and (3.7), sufficiency follows from equalities (3.10) and (3.11).

Since the distance $\rho_{3}$ in the case under consideration in accordance with (2.2) is constant, then, as we see, in addition to the oscillatory motion of a body with mass $\mu_{3}$ there is also its rotational motion when $C_{2} \neq 0$.

\section{An Assertion on Boundedness of Symmetric Motions}

For our further goals, we use some results on the two-body problem presented in [9]. Those results will be applied to the case where the masses of bodies are equal. In the framework of this case, we use $r$ instead of $\rho_{12}$ and $r$ instead of $\rho_{12}$ respectively.

Let us write down the equation for $r$ in the form

$$
r^{2^{\prime \prime}}=2 v^{2}-\frac{2}{r}
$$

Where,

$$
v^{2}=r^{\prime 2}=r^{\prime 2}+\frac{\left|r \times r^{\prime}\right|^{2}}{r^{2}}=r^{\prime 2}+\frac{c^{* 2}}{r^{2}}
$$

The energy integral is represented as

$$
\mu^{2}\left(r^{\prime 2}-\frac{2}{r}\right)=2 \tilde{h}=\text { const }
$$

Next we use the known equalities for the two-body problem

$$
\begin{aligned}
& \frac{1}{r}=\frac{1}{c^{* 2}}(1+e \cos f), \\
& r^{\prime 2}=\frac{e^{2}}{c^{* 2}} \sin ^{2} f, \\
& v^{2}=\frac{1}{c^{* 2}}\left[1+e^{2}+2 e \cos f\right],
\end{aligned}
$$

Where the constant

$$
e=\sqrt{\frac{\mu^{2}+2 c^{* 2} \tilde{h}}{\mu^{2}}}
$$

is the eccentricity of the elliptical orbit, $f$ is a true anomaly. 
We recall some key definitions that we will use below.

Definition 1. We say that the motion $\rho(\tau)=\left(\rho_{1}, \rho_{2}, \rho_{3}\right)^{\tau}$ of system (1.1) is distal if the following inequality is satisfied:

$\left|\boldsymbol{\rho}_{i j}(\tau)\right| \geq c_{1} \quad \forall \tau \in R, \quad \forall i<j, 0<c_{1}=$ const

Definition 2. In accordance with [10], we say that a fixed pair of mass points $\left(\mu_{i}, \mu_{j}\right), i<j$, of system (1.1) is Hill stable if the following inequality is satisfied:

$\left|\boldsymbol{\rho}_{i j}(\tau)\right|<c_{2} \quad \forall \tau \in R, \quad 0<c_{2}=$ const

According to [6], if $h<0$ and $\left|C_{1}\right| \neq 0$, then the symmetric motions belonging to the manifold (2.1) are distal and, in addition, the pair of material points $(\mu, \mu)$ is Hill stable.

Assertion 2. Let $\rho(\tau)=\left(\rho_{1}, \rho_{2}, \rho_{3}\right)^{\tau}$ be a symmetric motion of system (2.1) belonging to the set $\Omega$.

Then, if

$\mu^{3}+c^{2} h \leq 0$,

Where

$c^{2}=\left|\boldsymbol{\rho}_{12} \times \rho_{12}^{\prime}\right|^{2}, c=$ const,

then the symmetric motion is bounded.

Proof. Further, the energy integral is conveniently written as

$\frac{1}{2 \mu}\left(\mu_{3} \rho_{3}^{\prime 2}+\mu^{2} \rho_{13}^{\prime 2}\right)-\frac{2 \mu^{2}}{\rho_{12}}-\frac{4 \mu \mu_{3}}{\rho_{13}}=2 h$

Rewriting it in the form

$\mu^{2}\left[\frac{1}{2 \mu}\left(\rho_{12}^{\prime 2}+\frac{\left|\rho_{12} \times \rho_{12}^{\prime}\right|^{2}}{\rho_{12}^{2}}\right)-\frac{2}{\rho_{12}}\right]+\frac{\mu_{3}}{2 \mu} \rho_{3}^{\prime 2}-4 \frac{\mu \mu_{3}}{\rho_{13}}=2 h$,

We consider it as a quadratic equation with respect to the value $1 / \rho_{12}$. As a result, we obtain

$\frac{c^{2} \mu}{2}\left(\frac{1}{\rho_{12}}\right)^{2}-2 \mu^{2} \frac{1}{\rho_{12}}+\left(\frac{\mu \rho_{12}^{\prime 2}}{2}+\frac{\mu_{3}}{2 \mu} \rho_{3}^{\prime 2}-4 \frac{\mu \mu_{3}}{\rho_{13}}-2 h\right)=0$

In view of (4.12), we have

$\frac{1}{\rho_{12}}=\frac{2 \mu}{c^{2}} \pm \frac{2}{c^{2} \sqrt{\mu}} \sqrt{\mu^{3}+c^{2} h-\frac{c^{2}}{2}\left(\frac{\mu \rho_{12}^{\prime 2}}{2}+\frac{\mu_{3}}{2 \mu} \rho_{3}^{\prime 2}-4 \frac{\mu \mu_{3}}{\rho_{13}}\right)}$

and the resulting equality is represented as

$$
\frac{1}{\rho_{12}}-\frac{2 \mu}{c^{2}}= \pm \frac{2}{c^{2} \sqrt{\mu}} \sqrt{\mu^{3}+c^{2} h-\frac{c^{2}}{2}\left(\frac{\mu \rho_{12}^{\prime 2}}{2}+\frac{\mu_{3}}{2 \mu} \rho_{3}^{\prime 2}-4 \frac{\mu \mu_{3}}{\rho_{13}}\right)}
$$

Under the conditions of the assertion 2, the left-hand side of equality (4.14) is always a real number.

Suppose now that the considered symmetric motion $\rho(\tau)=\left(\rho_{1}, \rho_{2}, \rho_{3}\right)^{\tau}$ is not bounded under the conditions of the assertion. Then there exists a sequence $\left\{\tau_{k}\right\}, k=1,2,3, \ldots$, such that

$$
\lim _{k \rightarrow \infty} \tau_{k}=\infty, \varlimsup_{k \rightarrow \infty} \rho_{13}\left(\tau_{k}\right)=\infty, \rho_{13}\left(\tau_{k}\right)=\left|\boldsymbol{\rho}_{13}\left(\tau_{k}\right)\right| \text {. }
$$

We first consider the case of condition (4.8), when the strict inequality 


$$
\mu^{3}+c^{2} h<0
$$

holds. Then, in accordance with (4.15), for the sequence $\left\{\tau_{k}\right\}$, there exists a sufficiently large number $k$ such that $1 / \rho_{13}\left(\tau_{k}\right)$ becomes an arbitrarily small number, and, as a result, the right-hand side of equality (4.14) becomes imaginary. We get a contradiction.

Let now

$$
\mu^{3}+c^{2} h=0
$$

Then, if we assume that motion is unbounded in this case, then (4.15) holds, and, consequently, in accordance with equations (2.1), $\rho_{12}(\tau)$ approaches an elliptic Keplerian motion as $k \rightarrow \infty$ And then, taking into account (3.5), we see that the term $\rho_{12}^{\prime 2}$ under the sign of radical in (4.14), which is associated with the pair $(\mu, \mu)$, is representable in the following form:

$$
\rho_{12}^{\prime 2}=\frac{\tilde{e}^{2}}{\tilde{c}^{2}} \sin ^{2} \tilde{f}
$$

Where $\tilde{e}, \tilde{c}$ and $\tilde{f}=\tilde{f}(\tau)$ have the same meaning as $e, c^{*}$ and $f$ in equality (3.5).

Since for the elements of the sequence $\left\{\tau_{k}\right\}$ we have

$$
\frac{1}{\rho_{13}\left(\tau_{k}\right)} \rightarrow 0
$$

as $k \rightarrow \infty$, then the limit expression for the sum of terms under the sign of radical in (4.14) takes the form

$$
\left\{-\frac{c^{2}}{2}\left(\frac{\mu \rho_{12}^{\prime 2}}{2}+\frac{\mu_{3}}{2 \mu} \rho_{3}^{\prime 2}-4 \frac{\mu \mu_{3}}{\rho_{13}}\right)\right\}_{\infty}=-c^{2} \frac{\mu}{4}\left\{\frac{\tilde{e}^{2}}{\tilde{c}^{2}} \sin ^{2} \tilde{f}+\frac{\mu_{3}}{\mu^{2}} \rho_{3}^{\prime 2}\right\}
$$

We now consider equality (4.18) in more detail. The function $\sin ^{2} \tilde{f}$ in its right-hand side is equal to one for $\tilde{f}=(2 i+1) \pi / 2, i=0,1,2, \ldots$, and since we study the motions belonging to the set $\Omega$, the right-hand side of equality (4.18) becomes negative for $\sin ^{2} \tilde{f}=1$.

As noted above, the pair of material points $(\mu, \mu)$, is Hill stable on manifold (2.1) and the motion in question is distal, which makes the velocities of the material points of system (2.1) limited. The period of the elliptic Keplerian motion approaching $\rho_{12}\left(\tau_{k}\right)$ as $k \rightarrow \infty$ is finite. Within this period, the function $\sin ^{2} \tilde{f}$, being continuous, takes all its values. Thus, taking into account (4.17) and (4.18), we get every reason to assert that there exists a value $\tau^{*}(k)$ such that

$$
\left[-\frac{c^{2}}{2}\left(\frac{\mu \rho_{12}^{\prime 2}}{2}+\frac{\mu_{3}}{2 \mu} \rho_{3}^{\prime 2}-4 \frac{\mu \mu_{3}}{\rho_{13}}\right)\right]_{\tau=\tau^{*}(k)}
$$

becomes negative and, as a result, the right-hand side of equality (4.14) becomes imaginary. As we noted above, under the conditions of the assertion 2, the left-hand side of equality (4.14) is always valid. We arrive at a contradiction, whence we conclude that the assertion 2 is true.

As it is implied by the scheme of proof of assertion 2, boundedness of symmetrical motions remains to be true also in the case when the constant $\mu^{3}+c^{2} h$ is positive, but is quite small.

So, if the oscillating motions exist, then they can be realized only if the condition: $\mu^{3}+c^{2} h>\delta$ is satisfied, where $\delta$ is a small positive number. This fact may be of some practical interest.

\section{Conclusion}

In our proof of the assertion 2 on boundedness of symmetric motions, a key point is the fact that in case of moving the third body to infinity, under the conditions of the assertion 2 , the pair $(\mu, \mu)$ ac- 
quires the properties of an elliptic Keplerian motion. Moreover, as follows from the proofs of the assertions 1 and 2, the boundedness of symmetric motions is essentially associated with the absolute value of the angular momentum of the pair $(\mu, \mu)$ and the constant of the energy integral $h$, as well as with their relation.

\section{References}

1. KA Sitnikov (1960) The existence of oscillatory motions in the three-body problem. Dokl Akad Nauk SSSR 133: 303-306.

2. VM Alexeev (2001) Lecture on the celestial mechanics. Moscow-Izhevsk, R\&C.

3. J Moser (1973) Stable and random motion in dynamical system. Princeton: Princeton University Press, 216.

4. C Marchal (1990) The three - body problem. Oxford, Elsevier.
5. J Chazy (1922) Sur l'allure finale mouvement dans le problème des trois corps quand le temps croitindèfiniment. Ann I'Ecole Norm Supèr, 3ème Sèr 39: 29-130.

6. SP Sosnitskii (2014) On the bounded symmetrical motions in the three-body problem. Int Journ of Non-Linear Mech 67: 34-38.

7. SP Sosnitskii (2008) On the orbital stability of triangular lagrangian motions in the three-body problem. Astron J 136: 2533-2540.

8. SP Sosnitskii (2019) On an analogy in the restricted and general three-body problems. Adv Space Res 64: 1160-1165.

9. AE Roy (1978) Orbital Motion. Adam Hilger LTD, Bristol England.

10.VG Golubev, EA Grebenikov (1985) The three-body problem in the celestial mechanics. Univ Publ Moscow. 